\title{
Nuclear Magnetic Resonance Study of Poly(oxymethylene)
}

\author{
Kunio Hikichi, Akihiro Tsutsumi, Jun'ichi Oda, and Motozo Kaneko \\ Department of Polymer Science, Faculty of Science, \\ Hokkaido University, Sapporo, Japan.
}

(Received August 8, 1973)

\begin{abstract}
NMR measurements were carried out for poly(oxymethylene) samples which were subjected to various kinds of treatment such as annealing, rolling, and $\gamma$ irradiation. It was found that the narrow component narrows in two steps and that the line narrowing which appeared at higher temperatures is markedly influenced by sample treatment. The second moment of amorphous regions at liquid-nitrogen temperature, $\left\langle\Delta H^{2}\right\rangle_{a}$, was estimated. The result indicates that the amorphous region of the quenched sample is heterogeneous. Annealing was found to transform the morphology to the more nearly ideal two-phase structure. The decrease of the second moment between -197 and $20^{\circ} \mathrm{C}$ is due to molecular motion in the crystalline region as well as the lattice expansion.

KEY WORDS Poly(oxymethylene) / NMR / Annealing / $\gamma$-Irradiation / Rolling / Second Moment / Two-Phase Model.
\end{abstract}

In recent years a considerable amount of work has been done to investigate molecular motion in semicrystalline polymers. An interpretation of molecular motion in semicrystalline polymers is, however, seriously complicated by the rather complex morphology of these samples. An obvious approach to this problem is a systematic variation of morphological structure (degree of the crystallinity, crystallite thickness, and so on) and the study of the resulting effects with regard to molecular motion. This has been done for polyethylene by Olf and Peterlin ${ }^{1}$ using the nuclear-magnetic-resonance (NMR) method and by many authors by other relaxation methods. ${ }^{2}$ No such approach to poly(oxymethylene) (POM) has yet been reported.

In this paper, we present results of NMR measurement for POM samples which were subjected to various kinds of treatment such as annealing, rolling, and $\gamma$-irradiation. The results are discussed in terms of the two-phase model of semicrystalline polymers.

\section{EXPERIMENTAL}

The material studied was Delrin 150X manufactured by E.I. du Pont de Nemours \& Co. Sheets of about $0.5-\mathrm{mm}$ thickness were prepared by compression-molding at a temperature of $180^{\circ} \mathrm{C}$ to eliminate influence of prior history. After molding, the hot molding platen was immersed into cold water for quenching. Strips cut from a sheet were packed in glass tubes sealed off in vacuo for the use of NMR measurements.

Annealing was performed by holding the samples in evacuated glass tubes at temperatures of $100^{\circ} \mathrm{C}$ and $150^{\circ} \mathrm{C}$ for $10 \mathrm{hr}$ and at a temperature of $170^{\circ} \mathrm{C}$ for $5 \mathrm{hr}$, respectively. Parts of the sheet were $\gamma$-irradiated in air at room temperature to varying doses with a ${ }^{60} \mathrm{Co}$ source. One of the sheets was elongated by rolling to three times the original length at room temperature. The specification of samples used in this work is summarized in Table I.

The density of the samples was determined by a density gradient column of a mixture of xylene and carbon tetrachloride at a temperature of

Table I. Specification of samples

\begin{tabular}{cl}
\hline Sample & \multicolumn{1}{c}{ Specification } \\
\hline Q & Quenched from $180^{\circ} \mathrm{C}$ into cold water. \\
A1 & Annealed at $100^{\circ} \mathrm{C}$ for $10 \mathrm{hr}$. \\
A2 & Annealed at $150^{\circ} \mathrm{C}$ for $10 \mathrm{hr}$. \\
A3 & Annealed at $170^{\circ} \mathrm{C}$ for $5 \mathrm{hr}$. \\
I1 & $\gamma$-Irradiated at room temperature, $8 \times 10^{5} \mathrm{r}$. \\
I2 & $\gamma$-Irradiated at room temperature, $1 \times 10^{7} \mathrm{r}$. \\
R & Rolled to 3 fold at room temperature. \\
\hline
\end{tabular}


$28^{\circ} \mathrm{C}$. The degree of crystallinity $X_{\mathrm{c}}$ was determined from the density using the following equation

$$
1 / \rho=X_{\mathrm{c}} / \rho_{\mathrm{c}}+\left(1-X_{\mathrm{c}}\right) / \rho_{\mathrm{a}}
$$

where we use values of $1.51 \mathrm{~g} / \mathrm{cm}^{3}$ and $1.25 \mathrm{~g} /$ $\mathrm{cm}^{3}$ as densities of the crystalline, $\rho_{\mathrm{c}}$, and the amorphous region, $\rho_{\mathrm{a}}$, respectively. ${ }^{3,4}$

Wide-line NMR spectra were measured over a wide range of temperature. The apparatus and procedure have been reported elsewhere. ${ }^{5}$

\section{RESULTS AND DISCUSSION}

As has been reported in previous publications, ${ }^{6-10}$ spectra obtained for POM at room temperature consist of two components, broad and narrow; the latter becomes noticeable at a temperature of about $-50^{\circ} \mathrm{C}$.

The temperature dependence of the line width, $\Delta H$ (peak-to-peak separation in gauss) for POM samples is nearly the same as those reported previously. The broad component narrows in the temperature region from -90 to $-20^{\circ} \mathrm{C}$. It was found that the narrowing region of the broad component is little affected by sample treatments such as annealing, $\gamma$-irradiation, and rolling.

There is a very large decrease in line width of the narrow component with increasing temperature, which occurs essentially in two stepsone from 7 gauss to 3 gauss between -50 and $-20^{\circ} \mathrm{C}$, and the other to less than 1 gauss above $40^{\circ} \mathrm{C}$. The two-step narrowing of the narrow component has already found in data reported by Slichter. ${ }^{6}$

Figure 1 shows the variation of the line width of the narrow component as a function of temperature for the quenched sample $Q$ and the annealed samples A1, A2, and A3. Similar plots are shown in Figure 2 for the $\gamma$-irradiated samples I1 and I2, and the rolled sample R. In these figures data points are not plotted except for sample Q and I1 for the sake of clarity.

It is apparent that the narrowing of the narrow component at higher temperatures is markedly influenced by sample treatment. The effect of annealing and $\gamma$-irradiation is to move the narrowing region to lower temperatures, whereas the effect of rolling is to move the narrowing

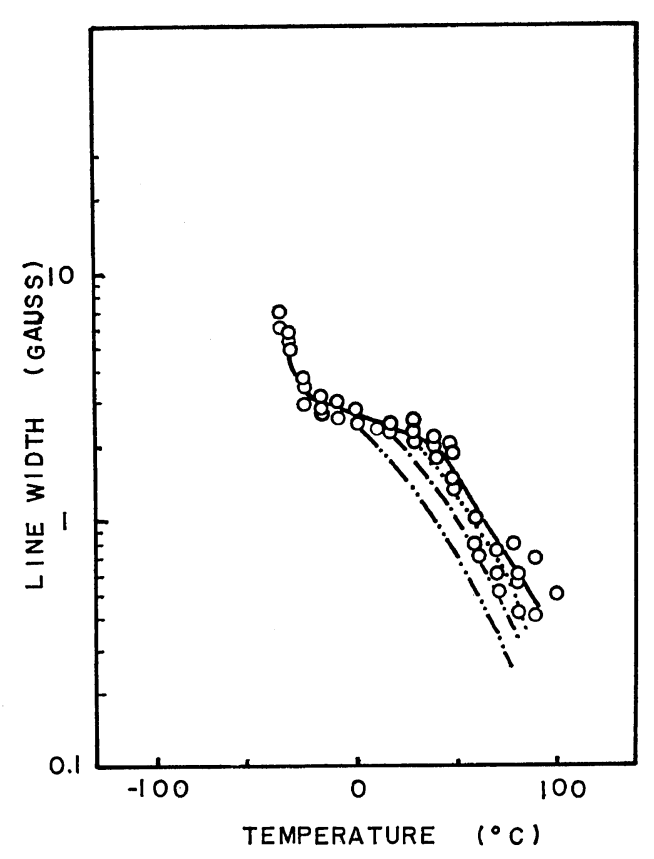

Figure 1. The line width of the narrow component as a function of temperature for poly(oxymethylene) samples: —, Q; $\cdots, \mathrm{A} 1 ;-\cdot-, \mathrm{A} 2 ;-\cdots, \mathrm{A} 3$.

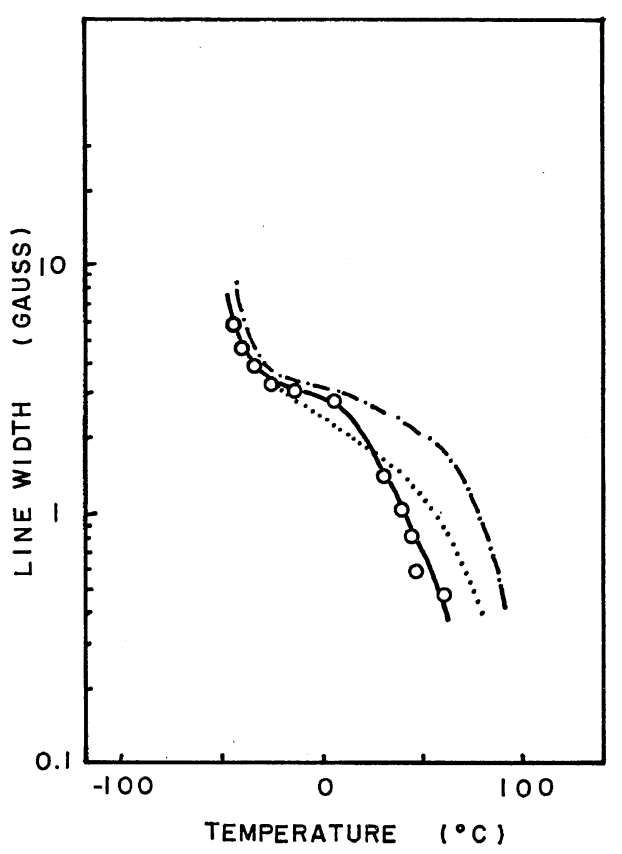

Figure 2. The line width of the narrow component as a function of temperature for poly(oxymethylene) samples: $-\mathrm{I} 1 ; \cdots, \mathrm{I} 2$; -.-, $\mathrm{R}$. 
Table II. The degree of crystallinity $X_{\mathrm{c}}$, the narrowing temperature $T_{\mathrm{N}}$, the second moments at liquid-nitrogen temperature $\left\langle\Delta H^{2}\right\rangle_{\mathrm{LNT}}$, and at room temperature

$\left\langle\Delta H^{2}\right\rangle_{\mathrm{RT}}$ and the difference between the two $\delta\left\langle\Delta H^{2}\right\rangle$

\begin{tabular}{cccccr}
\hline Sample & $X_{\mathrm{c}}, \%$ & $T_{\mathrm{N}},{ }^{\circ} \mathrm{C}$ & $\begin{array}{c}\left\langle\Delta H^{2}\right\rangle_{\mathrm{LNT}}, \\
\text { gauss }^{2}\end{array}$ & $\begin{array}{c}\left\langle\Delta H^{2}\right\rangle_{\mathrm{RT}}, \\
\text { gauss }^{2}\end{array}$ & $\begin{array}{r}\delta\left\langle\Delta H^{2}\right\rangle, \\
\text { gauss }^{2}\end{array}$ \\
\hline $\mathrm{Q}$ & 66.5 & 62 & 20.4 & 10.8 & 9.6 \\
$\mathrm{~A} 1$ & 68.8 & 60 & 19.6 & 10.3 & 9.3 \\
$\mathrm{~A} 2$ & 70.5 & 50 & 19.7 & 10.7 & 9.0 \\
A3 & 74.0 & 40 & 18.8 & 11.0 & 7.8 \\
I1 & 67.8 & 55 & 20.4 & 9.8 & 10.6 \\
I2 & 78.0 & 40 & 19.8 & 10.4 & 9.4 \\
R & 60.5 & 80 & 19.2 & 8.0 & 11.2 \\
\hline
\end{tabular}

region to higher temperatures. On the other hand, the narrowing of the narrow component at lower temperatures is found to be independent of sample treatment. In Table II the temperature $T_{\mathrm{N}}$, which is defined as the temperature where the line width becomes 1 gauss, is listed for each sample.

It has been well established that the polymer solid crystallized from the melt contains spherulite aggregates made of a stack of parallel lamellae in which the chain molecules are folded. There are some experimental data which unambiguously favor the location of all or of the larger part of the amorphous component on the two foldcontaining surfaces of the lamella. Iwayanagi, et al., carried out NMR measurements on the solid-state polymerized POM sample which is known to be completely crystalline. On the basis of the absence of the low-temperature narrowing from this kind of sample, they suggested that the chain folds and lamellar surfaces contribute to this narrowing. ${ }^{9-10}$ It is reasonable to assume that the narrow component is contributed from the amorphous layer on the fold-containing surfaces. Aoki, et al., ${ }^{4}$ reported from their small-angle X-ray scattering (SAXS) study of POM that the glass transition occurs at a temperature of $-60^{\circ} \mathrm{C}$. Miki, et al. ${ }^{11}$ suggested that the glass transition of POM is $-65^{\circ} \mathrm{C}$ on the basis of their dynamic mechanical and dielectric measurements. Thus, the appearance and the subsequent narrowing of the narrow component are due to segmental motion accompanied by the glass transition occurring in the amorphous layer. This kind of motion is found to be independent of sample treatment.

The fact that the narrow component narrows again at higher temperatures indicates that there must be an additional degree of freedom of movements in the amorphous layer on the lamella surface. Furthermore, the dependence of $T_{\mathrm{N}}$ on sample treatment suggests that this kind of movement of the chain in the amorphous layer is markedly restricted for the quenched and rolled samples and that the restriction is removed by annealing and $\gamma$-irradiation. The effect of $\gamma$ irradiation is thought to be two-fold, the chain scission and the temperature rise during the irradiation, which will show the same effect as annealing.

Aoki, et al., ${ }^{4}$ reported that there occurs an unexplained break point at a temperature of about $40^{\circ} \mathrm{C}$ in the plot of the square root of the relative intensity of the SAXS $v s$. temperature. This break point may be associated with the high-temperature narrowing of the narrow component. They suggested that the occurrence of this break point cannot be explained in terms of the density difference between the amorphous and crystalline regions.

Table II also indicates the second moment observed at liquid-nitrogen temperature, $\left\langle\Delta H^{2}\right\rangle_{\mathrm{LNT}}$, the second moment observed at room temperature $\left\langle\Delta H^{2}\right\rangle_{\mathrm{RT}}$ and the difference between the two, $\delta\left\langle\Delta H^{2}\right\rangle$.

In Figure 3 the decrease $\delta\left\langle\Delta H^{2}\right\rangle$ of the second moment between liquid nitrogen temperature and room temperature is shown for different samples, plotted $v s$. the respective noncrystalline mass fractions $\left(1-X_{\mathrm{c}}\right)$. The value at $\left(1-X_{\mathrm{c}}\right)=0$ was taken from data of the second moment of the 


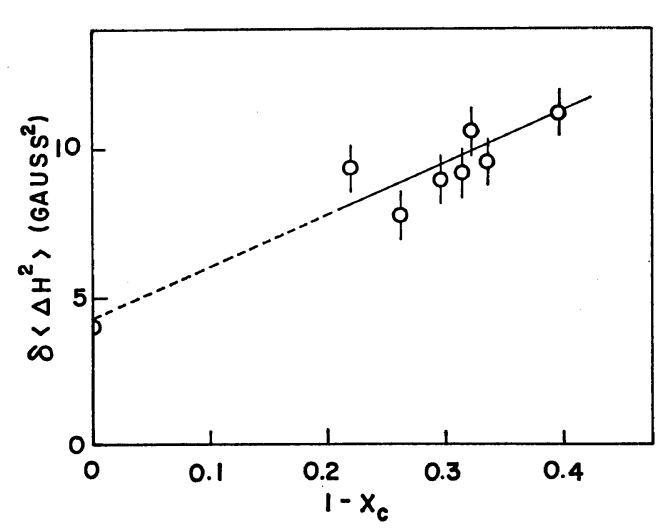

Figure 3. The decrease of the seeond moment, $\delta\left\langle\Delta H^{2}\right\rangle$, between liquid-nitrogen temperature and room temperature plotted as a function of the noncrystalline mass fraction $\left(1-X_{\mathrm{c}}\right)$. The data at $\left(1-X_{\mathrm{c}}\right)=0$ was taken from the ref 9 .

isotropic sample (randomly oriented needle-like crystals) reported by Iwayanagi, et al. ${ }^{9}$ As can be seen, the extrapolation of that line to 1 $\left.X_{\mathrm{c}}\right)=0$, i.e., to a fully crystalline sample, yields a second moment decrease of $4.2 \pm 1.7$ gauss $^{2}$.

The question arises as to what extent this decrease is due to molecular motion and, on the other hand, to the thermal lattice expansion. The distinction was made by calculating the second moment of a rigid POM lattice at $-196^{\circ} \mathrm{C}$ and at $20^{\circ} \mathrm{C}$, applying the hexagonal lattice constant at these temperatures. ${ }^{12}$ Values of $a=4.410 \AA$ at $-196^{\circ} \mathrm{C},{ }^{4}$ and $a=4.460 \AA$ at $20^{\circ} \mathrm{C}$ were used along with helical parameters given by Tadokoro, et al. ${ }^{13}$ For the computation the assumption was made that $\mathrm{C}-\mathrm{H}$ distance is $1.09 \AA, \mathrm{HCH}$ bond angle is tetrahedral, and two protons lie on a plane bisecting the angle OCO and perpendicular to the vector joining adjacent two oxygen atoms.

Molecular orientation of the helical poly(oxymethylene) molecule in the hexagonal crystal lattice has not been known until now. Thus the second moment was calculated as a function of molecular orientation. Theoretical second moment values of 20.0 gauss $^{2}$ at $20^{\circ} \mathrm{C}$ and 20.5 gauss $^{2}$ at $-197^{\circ} \mathrm{C}$ were obtained. Furthermore, it was found that the effect of molecular orientation upon the second moment is within \pm 0.5 gauss $^{2}$ at these temperatures. Thus the second moment decrease due to lattice expansion only is less than 1 gauss $^{2}$, which is smaller than the observed decrease of 4.2 gauss $^{2}$. The remaining contribution to second moment decrease is concluded to be due to molecular motion occurring in a crystalline region in the temperature region from -196 to $20^{\circ} \mathrm{C}$. We reported from X-ray studies that the molecule in the crystal undergoes rotational oscillation around the helical axis of the amplitude of $14^{\circ}$ at room temperature. ${ }^{14}$ On the basis of the alignment angle dependence of the second moment of the solid-state polymerized POM sample at $20^{\circ} \mathrm{C}$, Iwayanagi, et al., suggested that POM molecule in the crystal undergoes a rather complicated oscillation of the amplitude of $16^{\circ}$ including an oscillation around an axis perpendicular to the helical axis as well as the rotational oscillation around the helical axis. ${ }^{10}$ All these conclusions are in agreement with the present conclusion obtained for meltcrystallized POM samples. This is in contrast to the case of polyethylene of which $\delta\left\langle\Delta H^{2}\right\rangle$ between -196 and $20^{\circ} \mathrm{C}$ is interpreted in terms of lattice expansion only. ${ }^{1}$

As can be seen in Table II, the quenched sample has the largest value of the second moment at liquid-nitrogen temperature. Furthermore it was found that the annealing results in a decrease in the second moment at liquidnitrogen temperature.

If we assume a second moment value of $\left\langle\Delta H^{2}\right\rangle_{\mathrm{c}}=20.5$ gauss $^{2}$ for the fully crystalline rigid lattice, the rigid lattice value of the second moment of the amorphous regions $\left\langle\Delta H^{2}\right\rangle_{\mathrm{a}}$ may be calculated by the following equation,

$$
\left\langle\Delta H^{2}\right\rangle_{\text {obs }}=X_{\mathrm{c}}\left\langle\Delta H^{2}\right\rangle_{\mathrm{c}}+\left(1-X_{\mathrm{c}}\right)\left\langle\Delta H^{2}\right\rangle_{\mathrm{a}}
$$

where $\left\langle\Delta H^{2}\right\rangle_{\text {obs }}$ is the observed second moment. The assumption of $\left\langle\Delta H^{2}\right\rangle_{\mathrm{c}}=20.5$ may be justified by data of Iwayanagi, et al. ${ }^{9-10}$

The estimated value of $\left\langle\Delta H^{2}\right\rangle_{\mathrm{a}}$ for each sample is listed in Table III. This table shows that $\left\langle\Delta H^{2}\right\rangle_{\mathrm{a}}$ decreases with annealing. It is of interest to note that $\left\langle\Delta H^{2}\right\rangle_{\mathrm{a}}$ for the quenched sample is comparable to the fully crystalline rigid lattice value $\left\langle\Delta H^{2}\right\rangle_{\mathrm{c}}$. The sample $\mathrm{A} 3$, annealed at $170^{\circ} \mathrm{C}$, has the lowest value of $\left\langle\Delta H^{2}\right\rangle_{\mathrm{a}}=14.0$ gauss $^{2}$, which is close to the intramolecular second moment value of 13.4 gauss ${ }^{2}$.

These results indicate that the chains in the 
Table III. The rigid lattice value of the second moment of the amorphous region $\left\langle\Delta H^{2}\right\rangle_{\mathrm{a}}$ for (polyoxymethylene) samples

\begin{tabular}{cc}
\hline Sample & $\left\langle\Delta H^{2}\right\rangle_{\mathrm{a}}$, gauss $^{2}$ \\
\hline Q & 20.2 \\
A1 & 17.6 \\
A2 & 17.8 \\
A3 & 14.0 \\
I1 & 20.4 \\
I2 & 17.3 \\
R & 17.2 \\
\hline
\end{tabular}

amorphous layer of the quenched sample are more densely packed in some places and hence are more loosely packed in others to keep the density of the whole sample to a lower value. That is, the amorphous layer of the quenched sample is more or less heterogeneous. Annealing and $\gamma$-irradiation relax highly strained chains in the dense amorphous layer of the quenched sample, some of which crystallize, and remaining chains assume a more probable amorphous conformation. In this respect, it should be noted that a prolonged annealing gives the well-developed first and second diffraction maxima of SAXS. ${ }^{4}$ It is, therefore, concluded that the description of the morphology by the two-phase model becomes more adequate by annealing.

Acknowledgment. This work was financed by the Ministry of Education of Japan.

\section{REFERENCES}

1. H. G. Olf and A. Peterlin, Kolloid-Z. Z. Polym., 2157 (1967).

2. E. W. Fischer, H. Goddar, and W. Piesczek, Polym. Sci. Symp., 32, 149 (1971), and references cited in there.

3. C. F. Hammer, T. A. Koch, and J. F. Whitney, J. Appl. Polym. Sci., 1, 169 (1959).

4. Y. Aoki, A. Nobuta, A. Chiba, and M. Kaneko, Polymer J., 2502 (1971).

5. A. Tsutsumi, K. Hikichi, and M. Kaneko, Japan. J. Appl. Phys., 7, 577 (1968).

6. W. P. Slichter, Makromol. Chem., 34, 67 (1959).

7. H. G. Olf and A. Peterlin, J. Appl. Phys., 35, 3108 (1964).

8. K. Hikichi and J. Furuichi, J. Polym. Sci., A3, 3003 (1965).

9. S. Iwayanagi and I. Sakurai, J. Macromol. Sci.Phys., B3(2), 259 (1969).

10. (a) S. Iwayanagi, Kobunshi Kagaku (Chem. High Polymers) 27, 481 (1970), (b) S. Iwayanagi and T. Shibata, Rep. Progr. Polym. Phys. Japan, 13, 347 (1970).

11. K. Miki, S. Nakamura, and M. Kaneko, Kogyo Kagaku Zashi (J. Chem. Soc. Japan, Ind. Chem. Sect.), 73, 1566 (1970).

12. H. Tadokoro, T. Yasumoto, S. Murahashi, and I. Nitta, J. Polym. Sci., 44, 266 (1960).

13. Y. Takahashi and H. Tadokoro, Abstracts, 19th annual meeting of the Society of Polymer Science, Tokyo, May, 20, 1970, p 244.

14. A. Chiba, A. Hasegawa, K. Hikichi, and J. Furuichi, J. Phys. Soc., Japan, 21, 1777 (1966). 